\title{
Analysis and Modeling of New Energy Automobile's Data Acquisition and Processing System
}

\author{
Wang Yanning, Yu Xia
}

Guangdong University of Science \& Technology, Dongguan, Guangdong, 523083,china

\begin{abstract}
The data acquisition and processing system of new energy automobile is to design a complete experimental system that can realize the automation of the experiment; which can collect the effective data efficiently, accurately. Tt can also help the automotive engineers to learn the related performance of the automobile. Through the analysis of the business needs of the system, on the basis of it to combine with practice, the system function can be designed, making it module, through designing each module, they can be integrated, and finally realize the data acquisition and processing system.
\end{abstract}

Keywords: New energy automobile, Data acquisition and processing system, Modeling

\section{Introduction}

The production of new energy automobile can be said to be a very complex process, while ECU in the car, namely, the production of a special microcomputer controller can be regarded as the most important part of the car. Meanwhile, the development of new energy automobile in China is relatively backward, there is no corresponding data in many aspects. The automotive industry is significantly different from the other industries, it can not get the conclusions by means of theory guiding the practice. In most of time, the automobile can obtain the result through a large number of experimental data, learn how to set some of the relevant values in the automobile from a large number of experiments[1-3].

Therefore, starting from the perspective of application, I designed the data acquisition and processing system of new energy automobile, the data acquisition and processing system of new energy automobile can collect all data of the automobile during the running process more comprehensively. Then it can process according to the relevance of these data, which can make the performance of the automobile perform more directly, thus it can help automobile engineers to understand the operating conditions of the automobile in the current situation, which can also provide basic data for their research. Moreover, it can help automotive engineers to study their performance better[4]. At the same time, we also can establish database of recorders for different cars, so that we can contrast them during the process of development and research, so as to understand the advantages and disadvantages of the car that we want to produce.

\section{The Description of System Requirements}

The final target of designing the data acquisition and processing system of the new energy automobile is to analyze the effect on missions data under the condition of changing the specific data of automobile through collecting data at different speed, ECU data as well as the 
data of the emission instrument. However, in practical experiments, it is impossible for us to carry out experiment on real road with a large number of devices (such as an emission meter), thus we need a kind of simulation test bed to carry on the experiment.

As shown in Fig.1, what the system is required is to connect the related equipment that can simulate the test bed in the figure, so as to form a whole part, which can effectively help the automotive engineers to analyze data better.

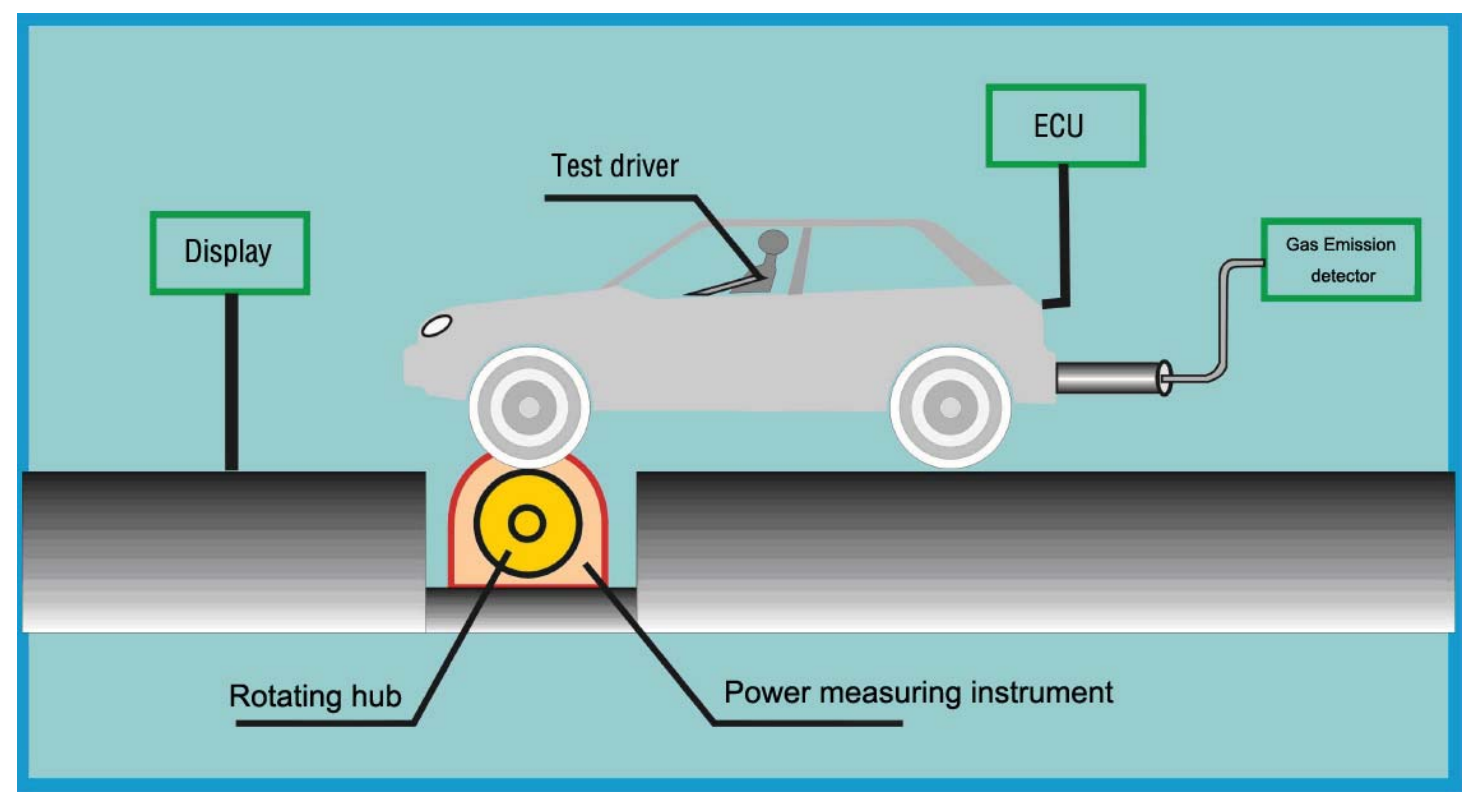

Fig. 1 Schematic Diagram of Simulation Test Bed of New Energy Automobile

\section{The Functional Requirements of System}

This system can start from the demand of automotive engineers, which can simulate the actual environment, on the indoor test bed, according to the predetermined program, it can carry on test in the specific data, in order to make sure the set of data, testing its performance, combustion efficiency, reliability and so on, so that we can learn the performance of the automobile under this circumstance. As shown in Fig. 1, what we need to do is to collect the data of automobile ECU as well as the data of emission meter in the simulation laboratory. The last thing we need to acquire is:

(1) In each simulation test, the corresponding data of $\mathrm{CO} 、 \mathrm{HC} 、 \mathrm{CO}_{2} 、 \mathrm{~A} / \mathrm{F} 、 \lambda 、 \mathrm{O}_{2}$ in the emission equipment should be simulated during the operation.

(2) In each simulation reality, simulating the operation of the ECU in the inlet pressure, battery voltage, inlet temperature, coolant temperature, solar term door position, before and after oxygen sensor, oxygen sensor, knock sensor, air temperature, air-fuel ratio, atmospheric pressure, injection correction value and injection pulse width and ignition advance the ignition angle, closure time, engine speed, target speed, idle idle valve position, BLM correction value, BLM unit, BLM learning value, intake pressure 2, vehicle speed, crankshaft tooth number, misfire rate, operation status, fault code, catalytic converter OSC, idle valve self value, mileage, operation time, charging efficiency rate, among thirty-two data, we can select part of data that we need, finally result can be derived.

\section{Non-functional Requirements of System}

The non-functional requirements of data acquisition and processing system for new 
energy automobile should include the following aspects:

(1) Security requirements

Due to the experimental data generated in the original car enterprises, the experiment data occurred during the process of having experiment should be its internal and confidential document, thus we should have certain requirement on the security of data system .

(2) Requirement on Performance

There are about three operators that can carry on the experiment of the system, according to the requirements of the automotive experiment, the frequency of using this system is expected to achieve ten times a month.The system is mainly used to save and export the relevant data in the process of the experiment, the total number of automobile will not exceed 100 kinds, while the result from the database will not exceed to forty. So the pressure of concurrency is not large, the system server requirement on system server is not so high.

(3) Requirement on Stability

During the process of having experiment, the system is operating in a continuous state, therefore, as for the system, the stability of the system is very important. Any faults of system can not affect the preservation and restoration of data, so the requires on higher hardware in this system is much higher, operating with high stability[5], meanwhile, meanwhile it must backup and restore database data timely.

(4) Requirement on Interface

The interface if the system is developed based on MFC, the interface is the user's first impression, so it requires the system to be enough beautiful and clean, friendly, so that the skin of the system can meet the aesthetic needs of the vast majority of users, which can make the user feel the system without a certain extent fatigue and improve work efficiency, thus the operation button of the system operation must be simple.

\section{The Analysis Model of System}

In this paper, the data acquisition and processing system of new energy automobile is analyzed and described, therefore we can see our goal is very simple, which is to collect ECU and emission measurement data of automobile in the specific automobile mechanical conditions, so as to make the engineers to have better analysis on the mechanical properties of automobiles. However, in the practical simulation test bed, the data that requires control and collection will be far more than these. For example, we can simulate the automobiles that are running on the actual road, then we need to install a hub on the automobile, with the application of a torque, so as to form a simulation of friction in the actual operation. Therefore, we will need a vast amount of data.

In this paper, the data acquisition and processing system of the new energy automobile is analyzed, it will set up the corresponding model from three dimensions, namely, function model, structure model and behavior model.

\section{The Functional Model of System}

We can analyze the function model of the system through the purpose of the experiment. As we know, the purpose of this system is to collect the data of EUC and emission meter during the process of simulating the real operation. The information that we need in the end is automobile information, the operating time of the automobile, the corresponding speed of the automobile, as well as the corresponding data of emission meter and ECU. 
If the automobile can be operated normally, we firstly need a fan system, which can simulate the real situation of the wind blowing of the automobile. We need to know the generated friction power on the experimental test bed, which should set the required speed in order to run, as well as the real speed under the premise of the friction conditions. Plus the formation of friction torque under the real condition.

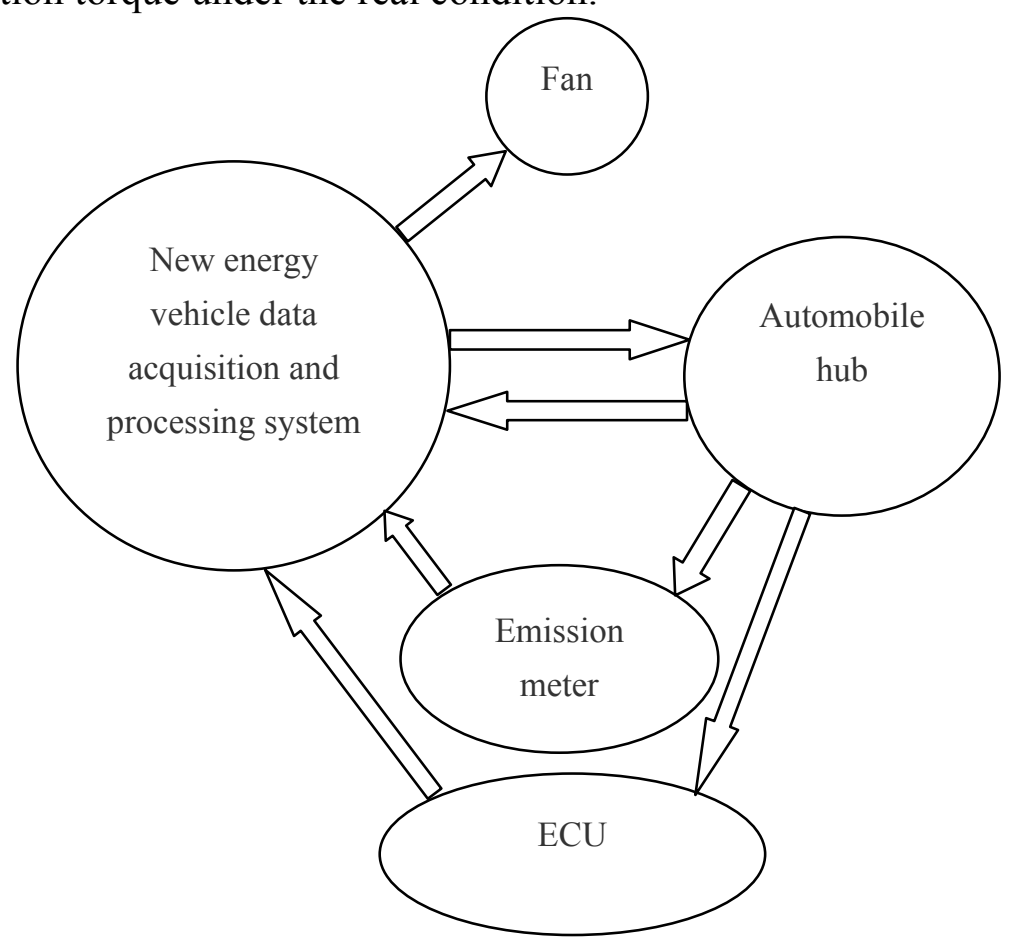

Fig.2 Schematic Diagram of Function Model of System

\section{Structure Model of System}

From the perspective of structure model to make analysis, we can say that the system can be divided into four parts in the form of substance experiments.

(1) Fan section. It is a one-way control of industrial computer over the fan, only the direction of the industrial computer transmitting information is in one-way direction. Including setting the position of opening and closing the fan as well as setting the gear.

(2) ECU part. This is in a one-way transmission direction to the industrial computer ECU. Only in the process of simulation experiment, ECU can send information to the industrial computer.

(3) Emission meter section. Like ECU, it is also in the simulation process, emission instrument will sent one-way information to the industrial computer.

(4) Hub section. With the industrial computer, they are in two-way transmission direction, including during the process of experiment, it can send the preset speed and torque information to the hub, moreover during the operation process, the real speed and torque information can be read out from the hub, so as to adjust it and carry out experiment in a much better way.

\section{Behavior Model of System}

This system, when we carry out simulation of road experiment, what we should do including: 
(1) The preset information of automobile torque. We need to know the torque of different automobiles at different speed, so that we can send the information to the hub in the process of simulation experiment, so as to make the corresponding torque.

(2) The information of presetting the simulation experimental condition. We need to preset the whole process of the automobile in the experiment, that is, V-T diagram of the simulation experiment of automobile.

(3) Set time for saving. The data that the instrument sent back to the industrial computer is continuously, then when we collect data, we need to set a time interval, so that we can get single data.

(4) Select the type of information. During the process of experiment, six groups of data of the emission instrument is all we need, however, ECU the thirty-two groups of data of EUC is not what we really want in the experiment for every time, therefore, it requires that we should have a selection process for the type of data.

\section{Reference}

[1] Juan, Zhang. "R\&D for environmental innovation and supportive policy: the implications for new energy automobile industry in China." Energy Procedia 5 (2011): 1003-1007.

[2] Yi, Nuo, and Guoyin Du. "The Social, Economic and Environmental Benefits Research of Mine Environment Governance Project [J]." Journal of Applied Science and Engineering Innovation 2.12 (2015): 477-480.

[3] Tsuchiya, Haruki, and Osamu Kobayashi. "Mass production cost of PEM fuel cell by learning curve." International Journal of Hydrogen Energy 29.10 (2004): 985-990.

[4] Ma X. Build the Structural Model of Risk Assessment on the AHP Method by Yaahp Software: a Case Study on Housing Fund[J]. Journal of Applied Science and Engineering Innovation, 2015, 2(6): 212-215.

[5] FANG H, Hu Y. An Analysis of the Tax Preferential Policy Effect for the Rapid Promotion of the New Energy Automobile [J]. Auto Mobile Science \& Technology, 2009, 3: 004. 